\title{
Design and Research of Crime Analysis and Early Warning System
}

\author{
Dong CAI* \\ Jiangsu Police Institute, Nanjing,Jiangsu 210031, China \\ *E-mail: tsaidong@126.com
}

\begin{abstract}
After the 21st century, social crimes were characterized by remoteness, high intelligence, and high technology. This paper combines the actual needs of prevention, carries out the overall planning and design of the crime analysis and early warning system, further learns the relational database technology and advanced network technology, proposes a fairly effective implementation plan, and promotes the informationization of crime early warning. The level has a very important practical guiding significance, which is conducive to reducing crime and thus maintaining public order.
\end{abstract}

KEYWORDS: wireless sensor networks, intelligent workshop products, moving target tracking, untraced Kalman filter

\section{Introduction}

With the continuous development of information technology, people's lifestyle has undergone great changes, and the Internet has become an important platform for people's information communication. Through this platform, people carry out economic activities such as e-commerce, online shopping, group purchase business, instant communication, online games, internet finance, etc. This new lifestyle brings convenience and quickness to people's life, production and social activities. However, the phenomenon of criminals using the network to carry out economic crimes has become increasingly prominent. According to related reports. Cybercrime is increasing at a rate of $30 \%$ per year, and more than $80 \%$ of websites worldwide have security vulnerabilities [1]. 


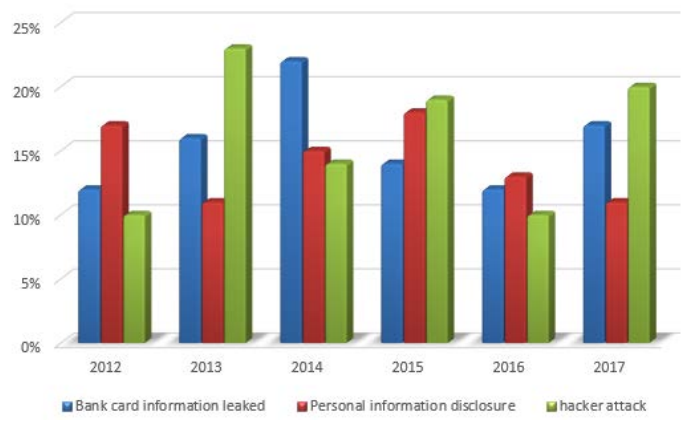

Figure 1 The growth trend of Internet crime

For the purpose of obtaining economic benefits, criminals use computers and communication networks to disrupt and disrupt networked terminals, endanger social and economic order and infringe on personal information, and violate relevant laws and regulations. In recent years, the growth trend of Internet crime shows in Figure 1. The essence of network economic crime is a new type of criminal form of traditional economic crime plus network technology, which is intelligent, complicated and high-tech. The database data was attacked by hackers, the bank card information and passwords were stolen by criminals, and the personal information of residents was leaked by unscrupulous personnel, which made the network economic crime intelligent, organized, professional and drooling. The series of features are becoming more and more prominent, and the traditional investigation mode is difficult to adapt to the need to combat crime. Therefore, in order to effectively combat the criminal activities of the Internet economy, in order to effectively combat the criminal activities of the network economy, it is necessary to change the concept of using modern information IT tools to carry out "online investigation", and build an overall intelligence and IT model for prevention and control and diversification [2]. The trend of change, to achieve the precise target and effectiveness of intelligence information detection, it can be seen that the prevention of cybercrime is an important way to mitigate and avoid the serious harm of cybercrime, strengthen the research and practice innovation of crime early warning work, and establish a "criminal warning The mechanism is an important issue placed in the research and practice of public security. 
Information is an important part of crime early warning work. The collection of early warning information can accurately and timely predict the signs of possible crimes, take effective measures to avoid and control the occurrence of illegal and criminal activities in advance, and kill criminal behavior in the cradle. The collection and extraction analysis of crime warning information is an effective means of crime prevention. By sorting, collecting data, and in-depth analysis of some of the collected early warning information, it is of great significance to extract some useful signal information for the search for police sources and warning signs.

\section{CRIME PREDICTION AND CRIME WARNING}

Crime prediction and early warning are concepts in criminology. In the field of crime prevention, crime prediction refers to the number of crimes and offenders obtained through investigations, statistics and other scientific methods of past and present criminal phenomena. And observe the signs that appear before the crime, and forecast and infer the future or unknown crime development trends, quantity increase and decrease, and species changes. Some scholars have also suggested that crime prediction refers to the estimation of the possible changes and development trends of criminal phenomena in a certain time and space in the future based on the correct understanding of the history and current situation of criminal phenomena and the law of its development and changes [3]. And judgment. Crime projections are also outlined as a practice that speculates on the overall trend, size, type, distribution, and individuality of future crimes by virtue of past facts related to crime and today's data. Crime early warning is a special way of crime prediction. It is the microscopic deepening of crime prediction by dynamically monitoring the development trend of crime phenomenon and capturing the signs of potential imbalance, disorder, and out of control.

The static structure diagram of the crime information early warning system reflects the relationship diagram of the system. The generation of the relationship diagram of the system requires the previous process. Generally, in the process of demand analysis, the domain model is generated through the demand analysis, and then generated. The domain model is refined on the basis of the domain model, and finally the relationship class diagram of the system is generated. In the criminal information early warning system, this paper adopts the layered design method, so 
that the extracted system core architecture can be more clear, design the whole system's class diagram as the first step, and then put the class diagram of each subsystem (package). In the distribution design, the class diagram of each use case is designed.

\section{Crime Warning Needs To Be Solved}

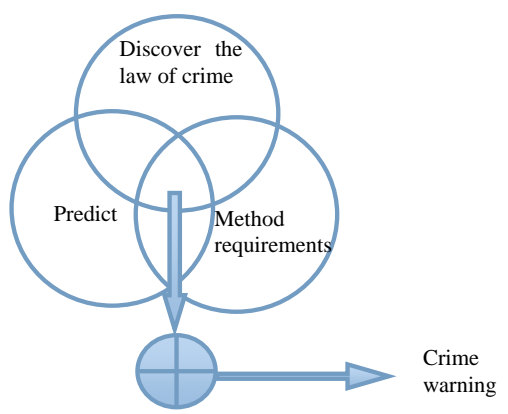

Figure 2 Crime analysis and early warning mechanism

Through the analysis of the characteristics of crime, we can see that there are certain rules in the occurrence of crime, but the law of value is not easy to be discovered, and it must be discovered through certain technical means. Therefore, this topic needs to find a means to discover the law of crime, and then use these rules to predict crimes, and use specific methods to give people a crime reminder to achieve crime warning. As we can see from Figure 2, to solve the crime early warning problem, we should first solve three problems:

\subsection{How to discover the law of crime}

Although there is no law in a single analysis of criminal activities, a large number of criminal activities can be concentrated to find out that there are certain laws. With the continuous development of the national public security information construction, the public security organs have accumulated a large number of criminal records, providing data support for solving the crime early warning problem. At the same time, data mining technology can quickly and accurately find 
valuable information from a large amount of data. Information can find hidden useful knowledge, change passive information into active information, and provide technical support for solving crime early warning problems [4].

\subsection{How to predict}

There are two ways to make a crime prediction: one is to predict the crime; the other is to make a crime prediction based on the user's location. The first method directly predicts the time and place of the crime, and helps the public security organs to predict the location of the crime in advance, which is conducive to guiding the public security organs' policing work and improving the efficiency of the public security organs. However, the first method does not prevent the occurrence of crimes. It can only help the public security organs to predict the occurrence of crimes, and the public security organs deploy police forces in advance, which is easy to be detected by criminals, leading to the time and place of criminals to change the crime or directly abandon the crime. In addition, the first way is difficult to give timely reminders to all those who may be involved in crime. The second way is to monitor the user's geographical location in real time, predict whether there will be crimes around the user, and make advance reminders, which is beneficial to the user to take effective preventive measures or stay away from crime, so that criminals can not commit crimes. Through a comparative analysis of the two approaches, data mining techniques can understand existing historical data and use it to predict future behavior. Use the reliable and stable forecasting function of data mining technology to predict crime. There are many ways to give people a crime reminder to give people a crime reminder. For example, the phone, SMS, mobile client, etc. can push the predicted crime warning information to the user.

\subsection{Method requirements}

The method to solve the crime early warning problem needs to meet at least the following six requirements:

Fast: Quickly respond to recent crimes, and prevent criminals from committing crimes; to ensure rapid forecasting, the predictive model can be downloaded to the client, and the predictive model of the client can be invoked when forecasting, while 
reducing server pressure. Timely: The forecast should be timely. When the user's position changes, the forecast should be made in time; the reminder should be timely, if the user is going to enter the crime occurrence area, give timely warning and reminder; ensure that the user can receive the early warning information in time, avoiding the possibility of crime. Location, to ensure personal and property security; timely update the client's forecasting model, set to download the latest forecasting model from the server at a random time in one day (according to the actual situation). It is foreseeable: predict the direction and distance of the user's travel, and give an early warning reminder before the user enters the crime occurrence area; simple: simple, convenient and easy to operate and universally applicable; safety: prevent use by criminals, prevent malicious damage, report cases, report fakes Cases and other phenomena; Accurate: The warning information must be accurate and credible, and there must be no reports of misinformation or misreporting, causing panic and affecting public order. Among them, the accuracy of the warning information depends on the accuracy of the historical criminal record used to analyze the law of crime. The more accurate the historical criminal record, the more correct the law is, and the more accurate the warning information. This requires the police to accurately record the case information, such as the location of the case. However, when the police record a case, they generally use the street, the area, etc. as a reference to confirm the location of the incident [5].

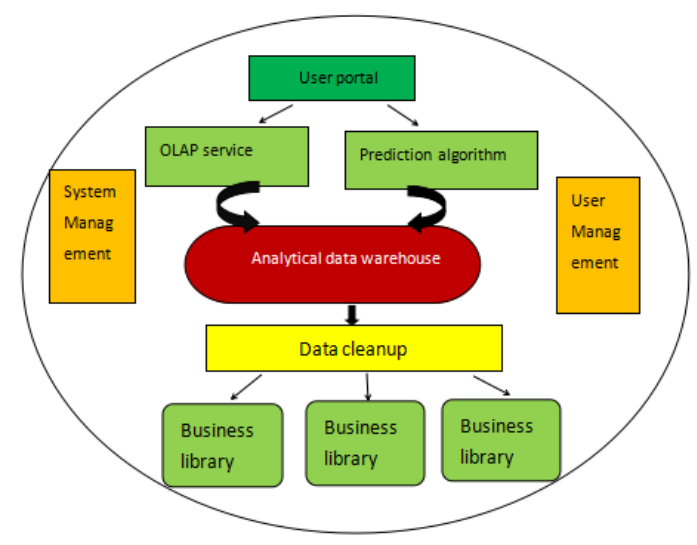

Figure 3 Meso-crisis prediction system architecture 
Although the streets and areas seem to be closely related to the occurrence of crimes, the regional streets will change with the development. For example, urban villages, road rectification, earthquakes, etc. will lead to changes in regional street division. Therefore, it is not reasonable to confirm that the crime location is based on the region, the street, etc., and the location where the crime is determined by latitude and longitude does not exist. The latitude and longitude is absolute and unique, and can be applied to various parts of the country.

\section{Design And Implementation of Crime Prediction And Early Warning System}

In the architecture of the meso-crisis prediction and warning system shown in Figure 3, in addition to the prediction algorithm, data warehouse, user portal, data cleaning, OLAP services, as well as user management, system management and many other aspects have mature system solutions. The core mesoscopic forecasting and warning algorithm has no clear public solution. Here, an implementation method of general prediction and early warning algorithm based on statistical probability is given. In this model, the collection of physical attributes of various predictive objects, such as regions, units, and time periods, is called different security subjects; various case types are divided into measurement basis, and various security subjects. The number of cases in a type case is a measured value.

First, through the public security business system (such as the 110 alarm system), collect a set of measured values (number of cases) representing the case of the security subject (sub-bureau, region, time period). Assume (C1, C2, ..., G). For each measured value ci $(1 \leq i \leq n)$, a range of measured values is established to classify this measured value $\mathrm{Ci}$, and this range of measured values is divided using $\mathrm{n}$ interval increments. Among them, the range of the last interval is the maximum value of the measured value to infinity.

After the system runs at intervals (such as 24 hours, weeks, quarters), the latest statistical (such as the last 30 days) statistical distribution of each specific measurement within the specified $\mathrm{n}$ intervals, that is, the first The relative frequency $\mathrm{P}$ in $\mathrm{M}$ intervals $(1 \leq \mathrm{M} \leq \mathrm{n})$. . At this point, there is a statistically based historical archive of all aspects of the system's behavior. The prediction is based on the latest (such as the last 30 days) measurement value and the statistical frequency, variance, 
and mean of the system, and the standard normal distribution of the probability density is obtained, so as to obtain the probability and interval of the specific case and the specific number of cases. Probability.

The alarm is to use the historical file based on statistics to judge the normal degree of each measurement $\mathrm{Ci}(1 \leq \mathrm{i} \leq \mathrm{n})$. Assume that the measured value $\mathrm{Ci}$ falls within the Lth interval $(1 \leq \mathrm{L} \leq \mathrm{n})$, and the maximum frequency it appears is set to the frequency of the interval in which the measured value $\mathrm{Ci}(1 \leq \mathrm{i} \leq \mathrm{n})$ and all the intervals in which the measured value is larger than $\mathrm{Ci}$. The sum of the frequencies

$$
T P R O B_{m}=Q_{m}+Q_{m+1}+\cdots+Q_{n}
$$

Use Wm indicating the degree of abnormality of the measured value $\mathrm{Ci}(1 \leq \mathrm{i} \leq \mathrm{n})$ and sets $\mathrm{Wm}$ the value of a variable that satisfies a mean of 0 and a variance of 1 normal distribution is greater than. s. The probability is equal to TPROBm. Value of Wm satisfies the equation

$$
Q\left(1 N(0,1) 1>W_{m}\right)=\text { TPROB }_{m}
$$

If TPROBm is $5 \%$, the system sets $\mathrm{Wm}=1.96$; if $\mathrm{TPROBm}=100 \%$, the system sets $\mathrm{Wm}$ is 0 . Also don't set Wm allowed greater than a specific value, so as to avoid there is a deviation in the calculation of the system behavior.

After processing a measured value $\mathrm{Ci}$, it is found that the $\mathrm{Ci}$ value is in the mth interval, and the characterization value of the current ci abnormality is set to be equal to Js. That is with TPROBm. Corresponding $\mid \mathrm{S}$ value. Assuming that there are n measurements for a particular system, then a statistical value U2 is used to characterize the degree of abnormality of the system, ie

$$
U^{2}=b_{1} S_{1}^{2}+b_{2} S_{2}^{2}+\cdots+b_{n} S_{n}^{2}
$$

In the formula, the port bi $(1 \leq \mathrm{i} \leq \mathrm{n})$ is a positive coefficient specified by the system administrator.

According to the definition of this $\mathrm{U}_{2}$ value, you can clearly see each value of a measured value $\mathrm{Si}$ contributed to the $\mathrm{U}_{2}$ value, and the system administrator

The corresponding measured value can be increased by increasing the value of the coefficient port; The importance of $\mathrm{Si}$. A larger $\mathrm{U}_{2}$ value will indicate the occurrence of anomalous behavior, while an U2 value close to zero indicates normal behavior (eg, behavior consistent with previously observed conditions). For the 
statistical value D, you can select one or more alarm settings (red, orange, yellow, green, etc.), these thresholds are connected to the level of abnormality of interest, when these abnormal levels are set, you can notify the system administrator. Since the statistical value $\mathrm{U} 2$ comprehensively reflects the user behavior in the most recent period, and the sequence of the $\mathrm{U} 2$ values are mutually dependent, the $\mathrm{U} 2$ value will slowly rise or fall. In order to avoid the generation of a continuous alarm signal, the system administrator can be notified only when the alarm status changes or after the alarm has been continued for a certain period of time (eliminating false alarms and continuous alarms).

Specifically, if the number of predicted cases needs to be performed, the probability of occurrence of the case number greater than a specific value may be predicted according to the simulated normal distribution; if the security situation analysis of the security principal (sub-bureau, region, time period) is performed, The measured data of various types (robbing, robbery, theft) are input as parameters to the calculation of the above-mentioned outliers, that is, the number of cases of various case types is used as a parameter; if a specific case type is carried out (robbing, robbery, theft) The security situation analysis can input the measured data of various security entities (sub-bureau, region, time period) as parameters into the calculation of the above-mentioned outliers, that is, the number of cases of various security entities is taken as a parameter.

\section{Conclusions}

The system adopts the system structure design, and provides a set of criminal information analysis and early warning system with simple operation, wide application and strong extensibility for the public security organs. It aims to improve the ability and efficiency of handling case-handling police cases and realize the information of criminal offenders. Management. The use of computer network technology makes it easier to handle cases. The main advantages of using public security network management are: on the one hand, the information of criminal offenders can be dynamically managed, and the modification, addition, and deletion can be realized in time, and it is unnecessary to prepare a huge archive as in the original, in a large number of documents. Finding data in a time-consuming and labor-intensive manner is extremely low-efficiency, and it also effectively avoids the 
possibility of these important work errors. On the other hand, the use of computer technology to manage these information resources is accurate, safe and simple. The system has the advantages of fast running speed, high safety, good stability and convenient maintenance, and can effectively improve work efficiency.

\section{Acknowledgements}

This work was financially supported by a Project Funded by the Priority Academic Program Development of Jiangsu Higher Education Institutions, the Theory and the Soft Science Project of Ministry of Public Security No. 2018LLYJJSST053, the Natural Science Foundation of Jiangsu Province No. BK20141033, the University Science Research Surface Project of Jiangsu Province No. 17KJB170005, Green seedling project of JSPI No. JSPI2018QM, Research and innovation team of JSPI No. 2018SJYTD12, the University Philosophy and Social Sciences Research Project of Jiangsu Province No. 2018SJA0465 and The Natural Science Project of JSPI No. 2017SJYZZ01.

\section{References}

[1]Waldo, G. P., \& Dinitz, S. (2016). Personality attributes of the criminal: an analysis of research studies, 1950-65. Journal of Research in Crime \& Delinquency, 4(2), 185-202.

[2]Kennedy, L. W., Caplan, J. M., Piza, E. L., \& Buccine-Schraeder, H. (2016). Vulnerability and exposure to crime: applying risk terrain modeling to the study of assault in chicago. Applied Spatial Analysis \& Policy, 9(4), 1-20.

[3]Matijosaitiene, I. (2016). Combination of cpted and space syntax for the analysis of crime. Safer Communities, 15(1), 49-62.

[4]Freisthler, B., Ponicki, W. R., Gaidus, A., \& Gruenewald, P. J. (2016). A micro temporal geospatial analysis of medical marijuana dispensaries and crime in long beach, california. Addiction, 111(6), 1027-1035.

[5]Yong, X. U., Zhang, X., Zhou, K., Wang, C., \& Xiaoren, X. U. (2017). Method and application of cause analysis for early-warning of resource and environmental system overloading. Progress in Geography, 36(3), 112-119. 